\title{
MECANISMOS ESTRATÉGICOS QUE FACILITARÁN LAS EXPORTACIONES DE LAS MYPE A LOS PAÍSES FIRMANTES DE LOS TRATADOS DE LIBRE COMERCIO - TLC PARA EL DESARROLLO DEL PAÍS
}

\author{
STRATEGIC MECHANISMS THAT WILL FACILITATE MYPE EXPORTS TO THE \\ COUNTRIES OF FREE TRADE AGREEMENTS - NAFTA FOR THE COUNTRY'S \\ DEVELOPMENT
}

\author{
Adrián Alejandro Flores Konja * \\ Docente Principal de la Facultad de Ciencias Contables, UNMSM
}

Manuel Alberto Hidalgo Tupia ${ }^{* *}$

Docente de la Facultad de Ciencias Contables, UNMSM

[Recepción: Abril de 2010 / Conformidad: Mayo de 2010]

\section{RESUMEN}

El artículo presenta las principales conclusiones y recomendaciones de la investigación del mismo tema y título, y que fue seleccionada por el Vicerrectorado de Investigación de nuestra Casa de Estudios. Además, se presentan los objetivos de la investigación, la metodología empleada y las diferentes situaciones que emergieron durante la investigación. La investigación utilizó fuentes primarias, recogidas mediante encuestas aplicadas con la ayuda de un grupo de alumnos de la Facultad a los gremios de MYPES, y también fuentes secundarias. La limitación fue la imposibilidad práctica de llegar aun sea indirectamente a las MYPES informales, obteniendo información directa solo de las MYPES formales más modernas y organizadas.

Como resultado de esta investigación, se pudo conocer que las MYPE no han usado el drawback, ni los demás mecanismos promotores de exportaciones, como un incentivo para internacionalizarse, debido a que las MYPES, en su gran mayoría, no se han formalizado. Entre las causas secundarias inmediatas, es que los directivos de las MYPE

\begin{abstract}
The article presents the main conclusions and recommendations of the research the same subject and title, and was selected by the Research of our House of Studies; in addition, we present the research objectives, methodology and the different situations that arose during the investigation. The research used primary sources, collected through surveys administered with the help of a group of students of the Faculty to the guilds of MYPES, and secondary sources too. The limitation was the impossibility practical of reach even if indirectly to the informal MSEs, obtaining direct information of the SMEs only formal, more modern and organized.

As a result of this investigation, it knows that the neitre the MYPES have not used the drawback, other mechanisms promoters of exports, as an incentive to internationalize, because the vast majority MYPES not been formalized. Secondary immediate causes, is that managers of MYPES are unaware of the benefits of the drawback of insufficient dissemination, which lack trained person-
\end{abstract}

* Doctor en Ciencias Contables y Empresariales, UNMSM. Contador Público Colegiado Certificado, actual Decano de la Facultad de Ciencias Contables, UNMSM. E-mail: afloreskonja@yahoo.es

** Economista, UNMSM. Docente de la Facultad de Ciencias Contables, UNMSM. E-mail: mhidalgot@hotmail.com 
desconocen las ventajas del drawback por insuficiente difusión; que carecen de personal capacitado para la aplicación del drawback; y carecen también de una adecuada estructura de costos para acogerse a este y demás regímenes e incentivos promocionales. Se concluyó en la necesidad que el Estado, debe apoyar a las MYPES a través de la construcción de infraestructura que reduzca los severos sobrecostos existentes y con medidas de abaratamiento del costo del dinero, a la par de un plan industrial, para propender a una verdadera competitividad nacional.

Palabras clave: Drawback. Exportaciones. Promoción de Exportaciones. MYPE. Formalización. Costos de exportación. Subsidio. Derechos Arancelarios. SUNAT. Régimen Aduanero.

\section{INTRODUCCIÓN}

La teoría económica considera que las exportaciones incrementan el ingreso nacional (representado por el PBI), y que por tanto son beneficiosas al crecimiento económico y aún al desarrollo, siendo deseable que se acompañe con un proceso de complejización productiva, o industrialización. Este argumento ha servido de fundamento a la negociación y firma de los Tratados de Libre Comercio de nuestro país con EE.UU. y otros países. En este contexto, las expectativas están puestas en las MYPEs como alternativa de crecimiento económico y de absorción de empleo, y en especial, para viabilizar su expansión a los mercados externos; por lo que se debería promover a las MYPE y alentarlas a su internacionalización.

Por esa razón, el Estado y las organizaciones multilaterales han establecido divesos mecanismos de apoyo y promoción de las Exportaciones, como los regímenes aduaneros (Drawback, Reposición de Mercancías nel for implementation of the drawback, and also lack of adequate cost structure to follow this and other schemes of export promotions .The incentives concluded on the necessity that the state should support MYPES through building infrastructure that reduces the existing cost overruns and severe measures lowering the cost of money, along an industrial plan, to propose a true national competitiveness.

Key words: Drawback, Exports, Export Promotion, MYPES, Formalization, Export costs, Allowance, Customs Duties, SUNAT, Customs Regime. en Franquicia y Admisión Temporal), los créditos promocionales a las exportaciones y los seguros de crédito a la exportación, además de la simplificación aduanera, como ejemplifica Exportafacil. Sin embargo, la percepción previa de los investigadores es que estos mecanismos no están siendo aprovechados adecuadamente por las MYPE, como lo indica el hecho que la participación de este sector como generador de exportaciones es reducido, y no hay señales de un cambio de tendencia.

Por ello, consideramos importante conocer si los mecanismos de promoción de exportaciones son o no adecuadamente utilizados por las MYPES para iniciar o consolidar su internacionalización, y conocer en caso contrario cuales son las causas que impiden este fin.

Se analizaron principalmente las normas tributarias y aduaneras que establecen los mecanismos promocionales, como el drawback, sus requisitos y condiciones para su aplicación. Se analizaron también textos sobre marketing y comercio internacional, 
donde se fundamenta la aplicación de estos mecanismos y se discute su aplicación en distintos países. Además, se consulto en revistas, periódicos y sitios web las opiniones de los gremios empresariales y de expertos sobre la aplicación de estos mecanismos, la evaluación de los resultados de su aplicación y las propuestas de mejora, provenientes de las Cámaras de Comercio, Sociedad Nacional de Industrias y Asociaciones de Exportadores. También se consultó información, en especial, del Ministerio de Trabajo, sobre las MYPE y sus principales características y extensión.

Notamos la carencia de un enfoque a la vez concreto y global respecto a la aplicación de estos mecanismos por las MYPES en el país, lo que motivó la investigación presente.

Los objetivos de la investigación planteada fue el determinar, si los mecanismos de promoción a las exportaciones son o no adecuadamente utilizados por las MYPE en nuestro país, a fin del aprovechamiento de las oportunidades del TLC; determinar, si fuera el caso, las razones por las cuales la aplicación de estos mecanismos promocionales no impactan favorablemente en las MYPE, $y$, también, el establecimiento de una estructura de costos para determinación del Drawback y otros regímenes de beneficios en exportación.

\section{PROBLEMÁTICA EN LA APLICACIÓN DEL DRAWBACK PARA LAS MYPE}

Una característica saltante que hallamos es que la informalidad es la realidad mas presente en el tema de las MYPE. El número de empresas que existen en el Perú, es de 3'200,000, que se agrupan en grandes, medianas, pequeñas y micro. Las grandes y me- dianas, son el $2 \%$ y representan a 64,000 empresas. Las micro y pequeñas, son el $98 \%$ y representan a 3'136,000 empresas. Del 98\% que son MYPE, el 74\% que representan a 2'541,000 empresas, opera en la informalidad. Es decir, el $72.52 \%$ de las empresas peruanas son informales. $\mathrm{Y}$ una característica central de los mecanismos de promoción de exportaciones que administra la SUNAT, es la formalidad.

En razón de esta limitación de la investigación, debimos dividir nuestro universo en dos grandes segmentos: las MYPEs formales y las MYPEs informales, concentrando nuestro análisis de fuentes primarias a las primeras y de fuentes secundarias exclusivamente, por razones obvias, a las segundas. Sin embargo, hay muchas características comunes a ambas, y otras diferenciales, como vamos a señalar.

Las características y aplicación del drawback se reseñaron en el artículo: "El drawback como mecanismo de promoción de exportaciones. ¿Cómo mejorar su impacto en las MYPES?", publicado en la revista de Investigación Contable de nuestra Facultad, "Quipucamayoc", número Enero - Junio de 2009.

Un análisis preliminar de fuentes secundarias nos permitió determinar las siguientes características de la aplicación del drawback por los MYPE:

Las empresas exportadoras o potencialmente exportadoras,

Tienen un nivel de exportaciones que en muchos casos no llegan al mínimo (US 10,000) para acogerse al régimen. Es decir, si se trata de microexportación, la empresa no podría acogerse (sin embargo, si hace varias operaciones pueden ser acumulables)

Encuentran dificultades en planificar sus operaciones de exportación al carecer de estructuras de costos de exportación que 
contemplen los beneficios del Drawback; por lo que, no pueden saber los beneficios reales de exportar utilizando este beneficio.

Encuentran dificultades para acogerse al Drawback, debido a que no siendo muchas veces importadoras directas, sus proveedores no cuentan con la documentación ordenada de la importación de los insumos, o dicha información no es fidedigna.

Por esta causa, son multadas por la aplicación indebida del Drawback, en razón a la inconsistencia o falta de integridad en la información proporcionada por un proveedor, acarreando la sanción no directamente al proveedor, sino a la empresa exportadora.

\section{METODOLOGÍA Y RESULTADOS}

\section{Metodología empleada}

La metodología se centró en la recolección de información procedente de los gremios de las MYPE, en general, y exportadoras, en particular, a través de encuestas aplicadas a los directivos de los gremios MYPE, y contrastándola posteriormente con el análisis documentario.

La primera etapa, en la cual se efectuó la recopilación de datos, se realizó con la participación de los alumnos ya mencionados, después de lo cual se hizo el análisis de la información con la ayuda de los paquetes informáticos SPSS y Excel.

Como resultado, se ha determinado nuestras apreciaciones y en función a estas se formularon las conclusiones. Cabe destacar que se separó por las razones ya indicadas dos segmentos, de MYPE formales e informales.

\section{Análisis de los resultados}

1. ¿Cuál es el porcentaje aproximado de sus asociados que realizan exportaciones? (MYPES FORMALES)

Según las estimaciones de los directivos de los gremios:

$16.66 \%$

Tabla N.o 1. Muestra - Gremios MYPE

Representan a 30,383 MYPES, formales, que no es la totalidad, sino el sector más organizado

- Cámara PYME Callao - CAPYMEC

- Confederación Nacional de Gremios de la Micro y Pequeña Empresa del Perú - CONAMYPE

- Asociación Red Empresarial del Parque Industrial Infantas

- Asociación REDPYMES

- Central de Asociaciones Empresariales y Empresarios de la Micro y Pequeña Empresa del Cono Sur de Lima - APEMIVES

- Federación de Parques Industriales del Perú - FENAPIP

- Sociedad Nacional de Industrias - Comité de la Pequeña Industria - COPEI

- Asociación de Talleres y Empresas de Metal Mecánica del Perú - ATEM PERÚ

- Asociación de Pequeños y Medianos Industriales del Perú - APEMIPE

- Asociación de Fabricantes de Calzado, Artículos Afines y Complementarios - APEMEFAC

- Asociación de la Micro y Pequeña Industria Confeccionista de Gamarra - AMPIC

- Federación de Empresarios de Gamarra Perú - FEDEGA PERÚ

- Sociedad Nacional de PYMES 
Este resultado da cuenta que aún las MYPEs formales (solo el 16.6\%) están escasamente internacionalizadas, lo que coincide con los resultados obtenidos por el Ministerio de Trabajo en años anteriores.
Las MYPES informales no tiene la posibilidad de la internacionalización.

4. ¿Cuándo sus asociados exportan, cuál es el mecanismo de promoción de exportaciones que aplican? (MYPES formales).

\section{Gráfico N.o 1}

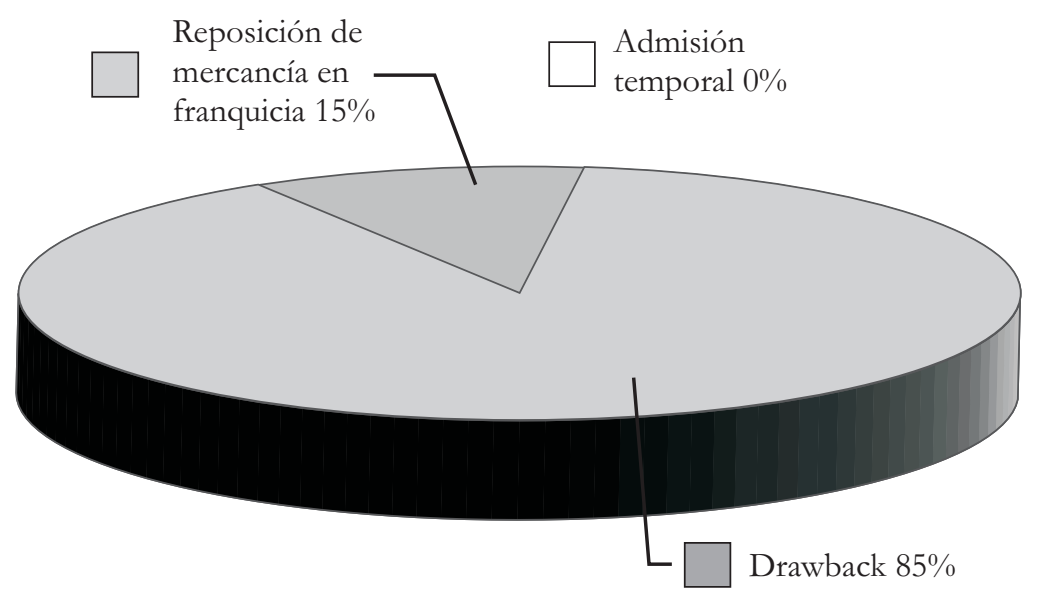

Este resultado muestra que el Drawback es el mecanismo o régimen promocional más usado por las MYPES formales.
5. Los directivos de las MYPE exportadoras (o potenciales exportadoras) de su gremio, iconocen los beneficios y requisitos del drawback? (MYPES formales).

\section{Gráfico N.o 2}

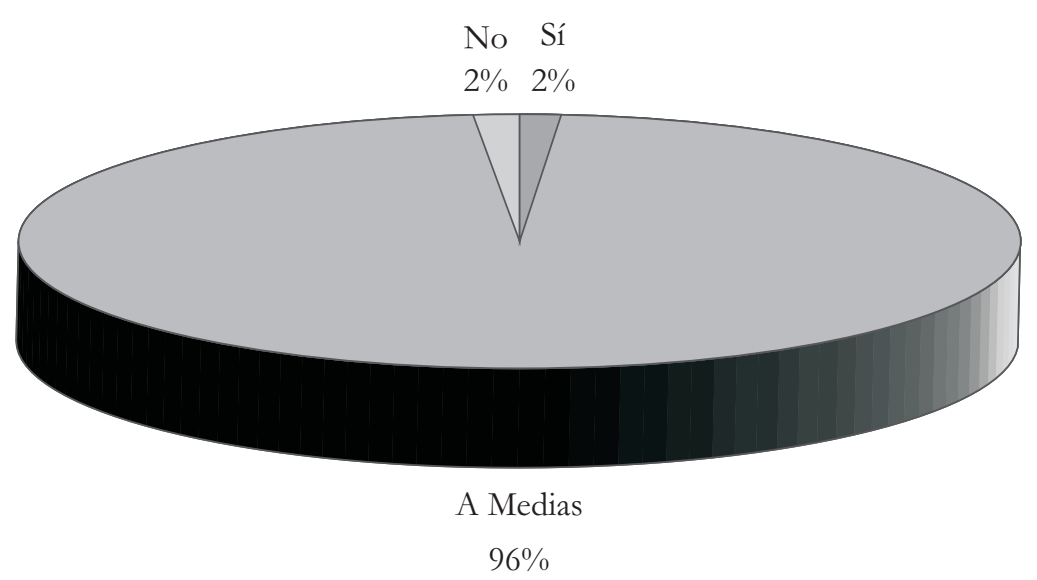

Este resultado permite reconocer que los propios conductores de las MYPEs (en un 96\%) tienen un conocimiento incompleto sobre el drawback.
6. Los directivos de las MYPE exportadoras (o potenciales exportadoras) de su gremio, ¿conocen cuales son los bienes que se pueden acoger al drawback? (MYPES formales). 


\section{Gráfico N.o 3}

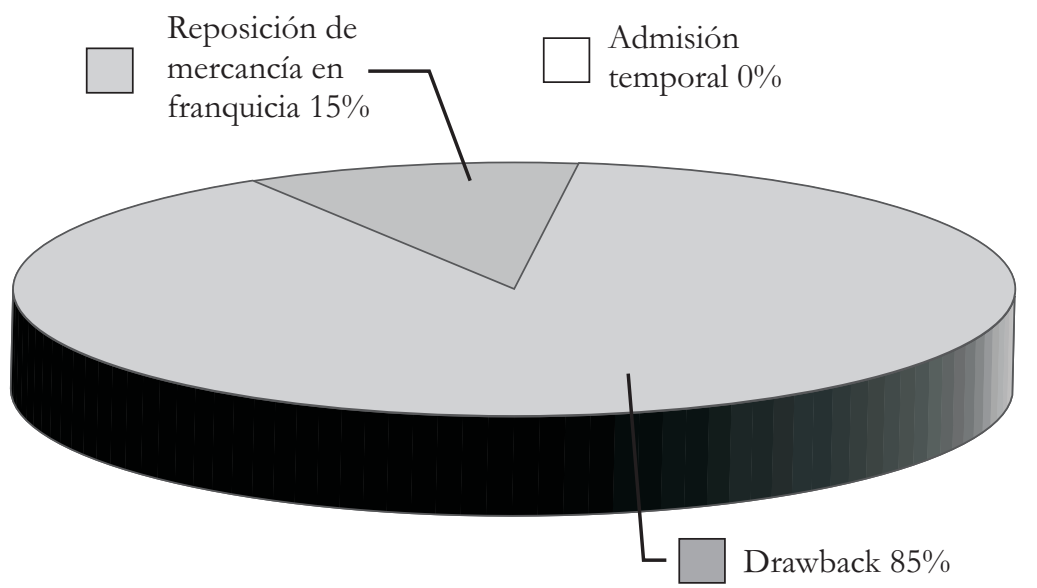

Estos resultados permiten reconocer que un 63\% de los propios conductores de las MYPEs desconocen que tipos de bienes pueden acogerse al drawback.
7. ¿Considera que las MYPE exportadoras (o potenciales exportadoras) de su gremio, tienen capacidad de gestión suficiente para aplicar el drawback y los demás regímenes aduaneros promocionales? (MYPES formales).

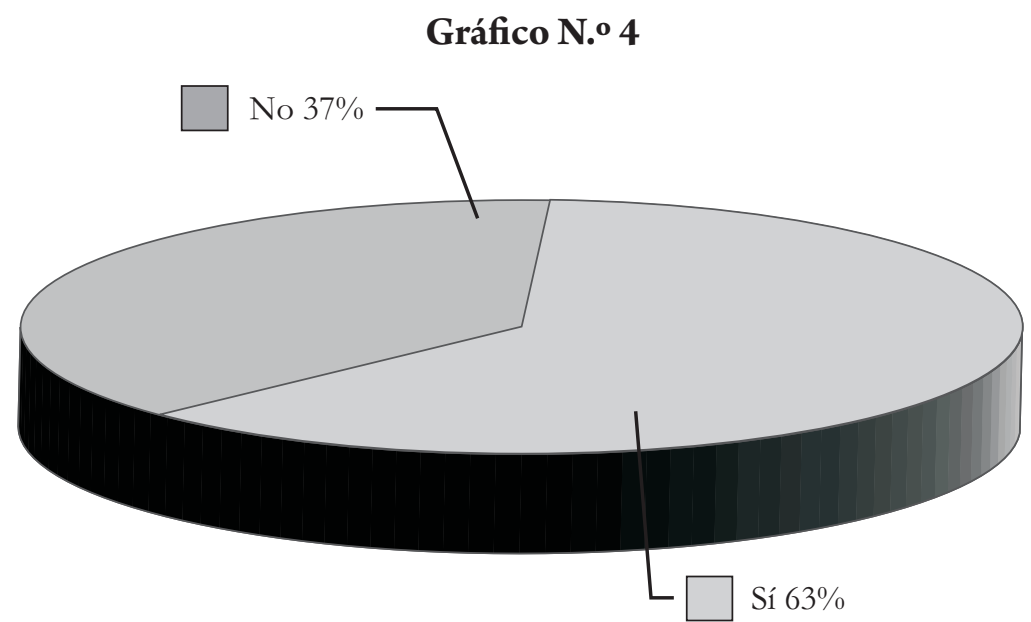

En este caso, la capacidad de gestión no se considera un obstáculo para el 63\% de los MYPEs formales para la aplicación de los regímenes de promoción de exportaciones.
8. ¿Considera que las MYPE exportadoras (o potenciales exportadoras) de su gremio, tienen o manejan una estructura de costos de exportación que les permita analizar los beneficios del drawback y los demás regímenes aduaneros promocionales? (MYPES formales). 


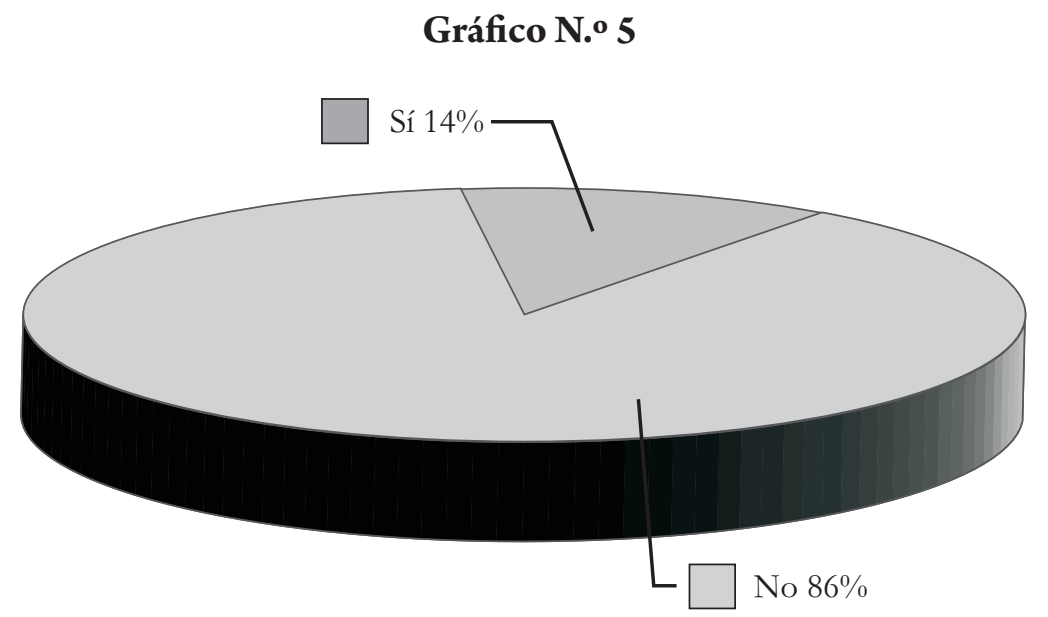

Estos resultados muestran que para un 86\% de las MYPEs formales carecen de una estructura de costos adecuada para una correcta aplicación del drawback y demás regímenes de promoción de exportaciones.

\section{CONCLUSIONES}

Las MYPE no se han beneficiado en el régimen de drawback u otros mecanismos promocionales, principalmente debido a su situación de informalidad.

Entre las causas inmediatas, establecidas provisionalmente, tenemos que los directivos de las MYPEs, exportadoras y potencialmente exportadoras, no conocen plenamente los beneficios y requisitos del drawback y demás mecanismos promocionales, carecen de capacidad de gestión para acogerse al régimen de Drawback y demás mecanismos promocionales .

Las empresas exportadoras o potencialmente exportadoras encuentran dificultades en planificar sus operaciones de exportación al carecer de estructuras de costos de exportación que contemplen los beneficios del drawback, por lo que no pueden saber los beneficios reales de exportar utilizando este beneficio.
Como causas concurrentes, se encuentran los sobrecostos en infraestructura y financiamiento. En infraestructura, la desventaja es evidente, en razón que al exportar un producto perecible desde el Callao a Miami puede ser hasta $15 \%$ más costoso que un flete para el mismo producto de Santiago de Chile a Miami. Movilizar un contenedor desde el puerto del Callao cuesta por todo concepto, aproximadamente U\$D 600, mientras que desde Valparaiso a Miami pueden costar hasta $30 \%$ menos. En Financiamiento, la MYPE tiene limitaciones de acceso en la banca formal, debido a que para acceder al mismo tiene que solventar tasas de 35 a $40 \%$ en nuestro país con lo cual nuestro producto no es competitivo en el mercado internacional.

\section{RECOMENDACIONES}

Debe promoverse mas activamente por el Estado la legalidad y la formalidad de las MYPE, que constituyen una obligación de todo empresario que, en suma, son una herramienta fundamental que nos permite ver y conocer lo que estamos haciendo como empresarios.

Debe promoverse tanto la investigación como la divulgación de los aspectos referidos al drawbacky otros mecanismos de promoción 
de exportaciones deben ampliarse, dada su gravitación para nuestra economía. La divulgación y capacitación a las MYPE de estos aspectos redundaría en una mejor y mayor aplicación del drawback y otros mecanismos de promoción de exportaciones por parte de las mismas.

Debe alentarse la responsabilidad de las propias empresas en relación a sus niveles de competitividad. Al respecto, existen serias deficiencias en cuanto a capacitación, costeos, negociaciones, formulaciones de planes de negocio e investigación de mercados representan algunos de los conceptos y herramientas de gestión pocos manejadas por las MYPEs y que son vitales para competir en mercados abiertos.

Se propone a las empresas la estructura de costos para la aplicación del drawback que figura en el Anexo N. ${ }^{\circ} 1$

Debe promocionarse la formación de Alianzas Estratégicas para las MYPE. Algunas MYPE tienen la posibilidad de exportar en forma directa. Algunas pueden exportar apelando a esquemas de asociatividad, como los consorcios. Otras MYPE, probablemente no exporten nunca, pero pueden participar en cadenas de exportación a través de sub contratas entre otras posibilidades de articulación empresarial. Las alianzas estratégicas significan una oportunidad para enfrentar la competencia.

El Estado debe procurar aplicar políticas para reducir los sobrecostos logísticos y financieros, cumpliendo su papel en la creación de infraestructura básica y generando políticas para abaratar el crédito a las MYPE.

\section{REFERENCIAS BIBLIOGRÁFÍCAS}

1. Cosio, F. (2007). Manual de Derecho Aduanero. Editorial RODHAS, 2. ${ }^{\text {a ed., }}$ Lima, Perú.
2. Cortez Tancun, Renee. El Drawback en el Perú 1995-2001. Tesis de Maestría. Facultad de Ciencias Contables. Lima 2002.

3. El Peruano, Ley General de Aduanas, aprobado mediante Decreto Legislativo N.o 1053, publicado el 27.06.2008; y su Reglamento aprobado con Decreto Supremo N.o 010-2008-EF, publicado el 16.01.2009.

4. El Peruano. Texto Único Ordenado de la Ley General de Aduanas, Decreto Supremo N.o 129-2004-EF, publicado el 12.09.2004; y su Reglamento, aprobado por Decreto Supremo N. ${ }^{\circ}$ 011-2005-EF publicado el 26.01.2005.

5. El Peruano. Tabla de Sanciones aplicables a las infracciones previstas en la Ley General de Aduanas, aprobada con Decreto Supremo N.o 031-2009-EF., publicado en El Peruano, el 11/02/2009

6. Flores Konja Adrián. Metodología de Gestión para las micro, pequeñas y medianas empresas en Lima Metropolitana”. Universidad Nacional Mayor de San Marcos, 2004.

7. Larios, Fernando. Flujos y Negociaciones Comerciales Agroalimentarias en el Perú . Asociación de Promoción Agraria. Konrad Adenauer Stiffung. Lima, agosto de 2002.

8. Ministerio de Trabajo y Promoción del Empleo. Dirección Nacional de la Micro y Pequeña Empresa. Consejo Nacional para el Desarrollo de la Micro y Pequeña Empresa. Actualización de Estadísticas de la Micro y Pequeña Empresa. Lima, Diciembre de 2007.

9. Palacios, L. (2009). ¿El drawback en el Perú es un subsidio? http://www.geocities.com/tacticaempresa/draw.doc (04/04/2009) 
10. Superintendencia Nacional Adjunta de Aduanas. Procedimiento General Restitución simplificada de Derechos Arancelarios. INTA PG 07. Publicado en El Peruano, 18/03/2009.

11. Sociedad de Comercio Exterior. Las multas del drawback. Semanario COMEXPERU del 20 al 26 de septiembre de 2004.

12. Sociedad Nacional de Industrias. Instituto de Estudios Económicos y Sociales. Reporte Macroeconómico. N.o 21, octubre de 2008.

13. Texto Unico Ordenado de la Ley de promoción de la competitividad, formalizacion y desarrolllo de la micro y pequeña empresa y del acceso al empleo decente. Ley MYPE.

14. Yacsahuache Monje, Ciro (2004) Análisis del financiamiento de las pequeñas y microempresas. Lima. CELA.

\section{ANEXO N.o 1}

\section{Estructura de costos para el control de drawback}

La determinación del valor base de cálculo para aplicar el $8 \%$ de restitución exige diseñar una estructura de costos donde se pueda distinguir aquellos elementos del costo que no deben formar parte de dicha base. La es- tructura tradicional de materiales, mano de obra y costo indirecto de producción no es suficiente para lograr una exitosa restitución por el mecanismo de Drawback. Esto en relación a los siguientes hechos:

- Debe conocerse el valor de los insumos importados usados; diferenciándolos de los nacionales;

- Debe determinarse si ya han recibido un beneficio arancelario precio, separándolos en dicho caso.

- Debe conocerse el valor total de los insumos empleados en relación al valor total de los exportado, pues Por otro lado, si el costo de las importaciones superan el $50 \%$ del FOB, no se tendría derecho a la restitución.

La siguiente estructura de costos fue propuesta por el profesor CPC Agustín Rodríguez Chávez, integrante del equipo de investigación, dentro del trabajo de investigación, quién aportó la casuística que presentamos a continuación, sobre la exportación de una turbina de generación de energía eléctrica. La primera estructura de costos (Cuadro N. ${ }^{\circ} 2$ ) no permite conocer ni declarar adecuadamente la base para aplicar el drawback, por lo que se ha modificado para ese fin en los dos cuadros siguientes (3 y 4), siendo el último usado para determinar si el insumo importado es mayor el 50\% al valor total de exportación. 
Cuadro N.o 2. Estructura de Costos - Tradicional

\begin{tabular}{|l|c|}
\hline $\begin{array}{c}\text { Exportación de } 1 \text { turbina generación de } \\
\text { energía eléctrica }\end{array}$ & $\mathbf{\$ 4 2 0 , 0 0 0}$ \\
\hline Materiales directos & 180,000 \\
\hline Mano de obra directa & 50,000 \\
\hline Costos indirectos de producción & 55,000 \\
\hline COSTO TOTAL & 285,000 \\
\hline Margen bruto & 135,000 \\
\hline Gastos de exportación & $-40,000$ \\
\hline Utilidad antes de participaciones e impuestos & $\$ 95,000$ \\
\hline
\end{tabular}

Cuadro N.o 3. Estructura de Costos - Propuesta cuadro

\begin{tabular}{|l|c|}
\hline $\begin{array}{c}\text { Exportación de } 1 \text { turbina generación de } \\
\text { energía eléctrica }\end{array}$ & $\mathbf{\$ 4 2 0 , 0 0 0}$ \\
\hline Materiales directos & 50,000 \\
\hline Material importado & 100,000 \\
\hline Material nacional & 30,000 \\
\hline Mano de obra directa & 50,000 \\
\hline Servicios de producción & 35,000 \\
\hline Costos indirectos de producción & 20,000 \\
\hline COSTO TOTAL & 285,000 \\
\hline Margen bruto & 135,000 \\
\hline Gastos de exportación & 40,000 \\
\hline Utilidad antes de participaciones e impuestos & $\$ 95,000$ \\
\hline
\end{tabular}


Cuadro N.o 4. Estructura de Costos - Valor exportación

\begin{tabular}{|c|c|c|c|c|}
\hline $\begin{array}{c}\text { Exportación de } 1 \text { turbina generación de } \\
\text { energía eléctrica }\end{array}$ & $\$ 420,000$ & $\$ 420,000$ & $\$ 420,000$ & $100 \%$ \\
\hline \multicolumn{5}{|l|}{ Materiales directos } \\
\hline Material importado & 50,000 & $12 \%$ & 10,000 & $2 \%$ \\
\hline Material nacional & 100,000 & $24 \%$ & 0 & $0 \%$ \\
\hline Mano importada proveedora local & 30,000 & $7 \%$ & 30,000 & $7 \%$ \\
\hline Mano de obra directa & 50,000 & $12 \%$ & & $0 \%$ \\
\hline Servicios de producción & 35,000 & $8 \%$ & & $0 \%$ \\
\hline Costos indirectos de producción & 20,000 & $5 \%$ & & \\
\hline COSTO TOTAL & 285,000 & $68 \%$ & 40,000 & $10 \%$ \\
\hline Margen bruto & 135,000 & $32 \%$ & & $0 \%$ \\
\hline Gastos de exportación & 40,000 & $10 \%$ & & $0 \%$ \\
\hline Utilidad antes de participaciones e impuestos & $\$ 95,000$ & $23 \%$ & & $0 \%$ \\
\hline Tipo de cambio utilizado & 2.948 & & & \\
\hline$\%$ de material importado & & $19 \%$ & & $0 \%$ \\
\hline Valor base de cálculo & & & 380,000 & $90 \%$ \\
\hline
\end{tabular}

Cuadro N.o 5. Cía. Metalmecánica SAC.

Catálogo de operaciones

\begin{tabular}{|r|l|c|c|}
\hline \multicolumn{1}{|c|}{ Cuenta } & \multicolumn{1}{|c|}{ Descripción } & Soles & Dólares \\
\hline 90 & Costo de materiales y componentes & & \\
\hline 901 & Costo de materiales & & \\
\hline 9011 & Costo de materiales nacional & & \\
\hline 9012 & Costo de materiales importado & & \\
\hline 9013 & Costo de materiales importado proveedor local & & \\
\hline 902 & Costo de suministros diversos & & \\
\hline 9021 & Costo de suministros diversos nacional & & \\
\hline 9022 & Costo de suministros diversos importado & & \\
\hline 9023 & Costo de suministros diversos importado proveedor local & & \\
\hline 906 & Envases embalajes de consumo directo & & \\
\hline 9061 & Envases embalaje consumo directo nacional & & \\
\hline 9062 & Envases embalaje consumo directo importado & & \\
\hline 9063 & Envases embalaje consumo directo importado proveedor local & & \\
\hline
\end{tabular}

En el Cuadro N. ${ }^{5}$, se propone una Estructura General de costos y Catálogo de operaciones para los fines a la aplicación del drawback y otros regímenes de promoción de exportaciones. 